\title{
Construct2 no Brasil: uma revisão sistemática de literatura
}

\author{
Hiago Oliveira de Jesus ${ }^{1}$, Almir de Oliveira Costa Junior ${ }^{1}$ \\ ${ }^{1}$ Universidade do Estado do Amazonas (UEA) - Escola Superior de Tecnologia (EST) \\ 69050-020 - Manaus - AM - Brasil. \\ \{hodj.lic, adjunior\}@uea.edu.br
}

\begin{abstract}
This article presents a systematic review of the Construct 2 use scenario in Brazil, identifying and analyzing journal articles published between 2013 and 2018. Through the Systematic Literature Review (RSL) it was possible to identify the public, the Construct use scenario 2 and research institutions that carry out studies with the tool and its methodologies. The RSL was motivated to measure the academic production in Brazil of the theme.
\end{abstract}

Resumo. Este artigo apresenta uma revisão sistemática sobre o cenário de utilização do Construct 2 no Brasil, identificando e analisando artigos de periódicos publicados entre 2013 e 2018. Através da Revisão Sistemática de Literatura (RSL) foi possível identificar o público, o cenário de utilização do Construct 2 e as instituições de pesquisa que realizam estudos com a ferramenta e suas metodologias. A RSL teve como motivação mensurar a produção acadêmica no Brasil sobre o tema.

\section{Introdução}

O Construct2 foi criado pela Scirra para o desenvolvimento de jogos baseado em Hyper Text Markup Language (HTML5). Os jogos produzidos nesta ferramenta, podem ser exportados para diversas plataformas e sistemas operacionais. A ferramenta é de código aberto, utiliza-se do Direct $X$ para reproduzir os jogos em navegadores de internet.

A ferramenta possibilita uma comunicação externa com uma base de dados, por meio da utilização do plug-in Ajax. Ele é responsável por realizar a troca de informações e a transferência de dados com diversas plataformas online por meio de scripts escritos na linguagem PHP. A integridade da troca de dados é mantida entre plataformas sob a gerência do MySql.

A Game Engine, permite produzir jogos que não exigem do usuário, uma experiência avançada de programação. A lógica de programação da ferramenta é baseada em comportamentos, permitindo a criação de jogos até por pessoas que não possuem conhecimentos específicos de programação. A programação é basicamente drag-and-drop de forma visual, seus componentes podem ser manuseados com ações de clicar, arrastar e soltar objetos.

Neste sentido, este artigo apresenta uma Revisão Sistemática da Literatura (RSL) com o objetivo de mensurar o cenário de produção acadêmica sobre o Construct 2 no Brasil. Para atingir este propósito, os resultados da RSL foram analisados a partir dos artigos publicados nos últimos 6 anos (2013-2018) no portal de publicações da Comissão Especial de Informática na Educação (CEIE). 
VIII Congresso Brasileiro de Informática na Educação (CBIE 2019)

Anais do XXV Workshop de Informática na Escola (WIE 2019)

As seções deste trabalho estão organizadas da seguinte maneira: a seção 2 apresenta o protocolo utilizado neste trabalho; a seção 3 descreve os resultados; a seção 4 apresenta as discussões em relação a revisão sistemática e por fim as considerações.

\section{Trabalhos relacionados}

$\mathrm{Na}$ literatura, é possível encontrar no contexto nacional, revisões sistemáticas que destacam a importância das ferramentas de criação de jogos e a programação visual.

Silva et al. (2014) realizaram uma investigação em periódicos internacionais para elaborarem uma revisão a respeito da validade da aplicação de jogos digitais no sentido de auxílio ao processo de ensino/aprendizado de programação. Scaico e Scaico (2016) desempenharam uma RSL para detectar e investigar pesquisas, a respeito da aplicação dos jogos em conteúdos curriculares de programação nos cursos superiores de Computação.

Considerando a necessidade de investigar-se o cenário de utilização do Construct 2, apresentamos uma revisão sistemática abrangendo os anos de 2013 a 2018.

\section{Materiais e métodos}

A RSL apresentada neste trabalho, foi elaborada utilizando o planejamento metodológico proposto por [Kietchenham, 2007]. Segundo o autor, o planejamento deve ser formado pela definição das questões de pesquisa, concepção da estratégia de busca e estruturação de critérios de exclusão, critérios de inclusão e critérios de avaliação.

O período de busca, levou em consideração trabalhos publicados no intervalo de $6 \operatorname{anos}(2013$ a 2018).

\subsection{Questões de pesquisa}

Esta RSL, utilizou-se da seguinte problemática norteadora: "Como o Construct 2 vêm sendo utilizado no Brasil?". Para responder essa pergunta, foram definidas as seguintes questões de pesquisa:

QP1: Quais os objetivos de uso do Construct 2 nas pesquisas expostas?

QP2: Quais os contextos e público-alvo mencionados?

QP3: Quais as estratégias de utilização da ferramenta?

QP4: Quais instituições de pesquisas envolvidas e sua localização no Brasil?

QP5: Quais as teorias mencionadas nos estudos?

\subsection{Estratégia de busca}

A estratégia de busca, foi conduzida através da utilização do Google Acadêmico como fonte de busca. Para isso, foram realizadas buscas com as palavras de busca "Construct 2 OR Scirra". Foram retornados 34 resultados de diferentes portais de publicações.

Neste primeiro momento, foram verificados os anais, eventos e revistas dos referidos artigos.

Os resultados obtidos, apresentam o portal de publicações da Comissão Especial de Informática na Educação (CEIE), como sendo o mais relevante (aquele com o maior número de publicações), apresentando: 2 artigos do Congresso Brasileiro de Informática na Educação (CBIE) e 1 do Simpósio Brasileiro de Informática na Educação (SBIE). 
VIII Congresso Brasileiro de Informática na Educação (CBIE 2019)

Anais do XXV Workshop de Informática na Escola (WIE 2019)

\subsection{Busca de artigos}

Os eventos e revistas identificados, disponibilizam mecanismos de busca próprios em suas bases. Ao utilizar as mesmas palavras de busca, pode-se identificar um número maior de resultados, se comparad o ao resultado obtido com o Google Acadêmico.

Deste modo, optou-se por realizar uma busca mais profunda diretamente no portal de publicação da CEIE utilizando seu mecanismo de busca. Nesta busca, foram encontrados 27 artigos distribuídos de acordo com a tabela 1.

Tabela 1. Número de artigos obtidos com a palavra de busca

\begin{tabular}{|c|c|c|c|c|}
\hline Bases & WIE & WCBIE & SBIE & RBIE \\
\hline Número de artigos encontrados & 8 & 3 & 12 & 4 \\
\hline
\end{tabular}

\subsection{Critérios de exclusão, inclusão e avaliação}

Em seguida, foi realizada a leitura dos títulos e dos resumos de cada um dos 27 artigos. Neste sentido, foram aplicados os critérios de exclusão e inclusão descritos na tabela 2.

\section{Tabela 2. Critérios de exclusão e inclusão}

\begin{tabular}{|l|l|}
\hline \multicolumn{1}{|c|}{ Critérios de exclusão } & \multicolumn{1}{|c|}{ Critérios de inclusão } \\
\hline E1. Artigos no formato de revisão sistemática. & I1. Artigos publicados entre 2013 e 2018. \\
E2. Artigos duplicados. & I2. Artigos com seis ou mais páginas. \\
E3. Artigos não relevantes (excluídos pelo título e resumo). & \\
E4. Artigos em idioma diferente de português. & \\
\hline
\end{tabular}

Por fim, foram utilizados os critérios de avaliação (Tabela 3. Esta etapa, tinha como objetivo, mensurar a importância dos artigos para responder as questões de pesquisa desta RSL.

Tabela 3. Critérios de avaliação

\begin{tabular}{|l|}
\hline \multicolumn{1}{|c|}{ Critérios de avaliação } \\
\hline A1. Qual o objetivo proposto do trabalho rela tado? \\
A2. Qual o público alvo do trabalho? \\
A3. Qual o ambiente de utilização? \\
A4. Quais os métodos/estra tégias de utilização da ferramenta? \\
A5. Quais as instituições de ensino superior (IES) envolvidas? \\
\hline
\end{tabular}

Neste sentido, realizou-se a leitura dos títulos e resumos e em alguns casos, foi necessário realizar a leitura completa dos 5 artigos.

\section{Resultados}

Na tabela 4 é apresentada uma descrição dos 5 artigos filtrados nesta RSL.

Tabela 4. Artigos incluídos na revisão

\begin{tabular}{|c|l|c|c|c|}
\hline ID & \multicolumn{1}{|c|}{ Título } & IES & Ano & Fonte \\
\hline 01 & $\begin{array}{l}\text { Robô Euroi: Estratégias Matemática para desenvolver o } \\
\text { Pensamento Computacional. }\end{array}$ & UEA & 2018 & WCBIE \\
\hline 02 & $\begin{array}{l}\text { Desenvolvimento de Jogos Digitais como uma Estratégia para } \\
\text { Despertar Novos Talentos: Um Relato de Experiência. }\end{array}$ & URI & 2018 & WIE \\
\hline 03 & $\begin{array}{l}\text { O livro do conhecimento: um jogo de aventura para exercitar a } \\
\text { ortografia. }\end{array}$ & $\begin{array}{l}\text { UEA, } \\
\text { UFAM }\end{array}$ & 2018 & SBIE \\
\hline
\end{tabular}


VIII Congresso Brasileiro de Informática na Educação (CBIE 2019)

Anais do XXV Workshop de Informática na Escola (WIE 2019)

\begin{tabular}{|c|l|c|c|c|}
\hline 04 & $\begin{array}{l}\text { Desenvolvendo a Programação de Jogos Digitais no Ensino } \\
\text { Médio: um Relato de Experiência Utilizando a Ferramenta } \\
\text { Construct } 2 .\end{array}$ & IFMA & 2017 & WCBIE \\
\hline 05 & $\begin{array}{l}\text { Uma Proposta de um Serious Game no Auxílio do Aprendizado } \\
\text { da Anatomia Humana. }\end{array}$ & UFPE & 2017 & SBIE \\
\hline
\end{tabular}

\subsection{QP1: Quais os objetivos de uso do Construct 2 nas pesquisas expostas?}

Em essência, os artigos apontam como objetivo, a utilização do Construct 2 para trabalhar o ensino de programação e o pensamento computacional por meio do desenvolvimento de jogos destinados à aprendizagem no Ensino Médio e Superior. A tabela 5 apresenta o detalhamento do objetivo de cada artigo.

Tabela 5. Objetivos dos artigos selecionados

\begin{tabular}{|c|l|}
\hline ID & \multicolumn{1}{|c|}{ Objetivo do artigo } \\
\hline 1 & Propõe um jogo para estimularo pensamento computacional atra vés de fundamentos da ma temática. \\
\hline 2 & $\begin{array}{l}\text { Propõe uma oficina de desenvolvimento de jogos com meninas do ensino médio na promoção do } \\
\text { pensamento computacionale o incentivo a Computação. }\end{array}$ \\
\hline 3 & $\begin{array}{l}\text { Propõe um jogo para estimular a aprendizagem da Língua Portuguesa atra vés de desafios de } \\
\text { ortografia. }\end{array}$ \\
\hline 4 & $\begin{array}{l}\text { Propõe uma oficina de programação com alunos do ensino médio para incentivara programaçã o de } \\
\text { jogos. }\end{array}$ \\
\hline 5 & Propõe um jogo de aprendizagem da anatomia humana no ensino superior. \\
\hline
\end{tabular}

\subsection{QP2: Quais os contexto e público-alvo mencionados?}

Embasados na utilização dos critérios A1, A2 e A3, foi possível descrever o cenário em que o Construct 2 foi utilizado. Dentre os cenários de uso, temos instituições de ensino básico, escolas públicas (ID.02 e ID.04), escola privada (ID.02) e universidade (ID.05). Quanto ao público-alvo envolvido, 2 com crianças e 3 com jovens e adultos (Ensino médio e Superior).

\subsection{QP3: Quais as estratégias de utilização da ferramenta?}

De modo geral, os artigos incluídos na RSL, apresentam o Construct 2 como uma ferramenta de suporte ao desenvolvimento de jogos educacionais (ID.01, ID.02, ID.03, ID.04, ID.05) e também como ferramenta para auxiliar no processo de ensino de programação e o incentivo à computação (ID.04).

\subsection{QP4: Quais instituições de pesquisas envolvidas e sua localização no Brasil?}

A RSL encontrou 5 instituições de pesquisas que desenvolvem estudos sobre o ensino de programação e o desenvolvimento de jogos. Dentre elas, a Universidade do Estado do Amazonas com dois estudos desenvolvidos nesta linha de pesquisa, sendo um artigo desenvolvido em parceria com a Universidade Federal do Amazonas. Além disso, alguns trabalhos foram desenvolvidos pelo Instituto Federal do Maranhão, Universidade Federal de Pernambuco, Universidade Regional Integrada do Alto Uruguai e das Missões. As regiões em que estes trabalhos foram desenvolvidos: $40 \%$ no Norte, $40 \%$ no Nordeste e $20 \%$ no Sul. 
VIII Congresso Brasileiro de Informática na Educação (CBIE 2019)

Anais do XXV Workshop de Informática na Escola (WIE 2019)

\subsection{QP5: Quais as teorias mencionadas nos estudos?}

As teorias de aprendizagem ou abordagem pedagógica mencionadas nos artigos são apresentadas na tabela 6. Apenas um dos artigos não mencionou ou apresentou a utilização de alguma teoria ou abordagem, no caso o artigo (ID.01).

Tabela 6. Teorias mencionadas nos artigos

\begin{tabular}{|l|l|}
\hline Teorias & Artigos \\
\hline Aprendizagem baseada em jogos & ID.02 \\
\hline Aprendizagem significativa & ID.05 \\
\hline Aprendizagem por descoberta & ID.04 \\
\hline Teoria cognitiva da aprendizagem multimídia & ID.03 \\
\hline Teoria da carga cognitiva & ID.03 \\
\hline
\end{tabular}

\section{Considerações finais}

Este estudo teve como propósito, desenvolver uma RSL sobre trabalhos referentes a utilização do Construct 2 no cenário nacional, levando em consideração artigos publicados nos últimos seis anos no portal de publicações da CEIE abrangendo os anos de 2013 a 2018.

Em essência, esta RSL pôde identificar uma tendência em utilizar o Construct 2 como estratégia para auxiliar o ensino de programação e estimular o pensamento computacional através da programação de jogos. Em relação ao cenário de utilização, os artigos analisados em sua grande maioria, concentram-se em alunos do Ensino Médio e uma minoria no Ensino Superior.

Pôde-se verificar que poucos estudos apresentam uma estratégia de avaliação dos seus estudos de caso. De certo modo, os artigos que mencionaram a metodologia de avaliação, aplicaram formulários, pré e pós intervenções. Observou-se ainda, uma baixa quantidade de abordagens pedagógicas nos artigos e pouco esclarecimento das metodologias utilizadas.

\section{Referências}

Eloy, Adelmo A. da Silva; LOPES, Roseli de D.; ANGELO, Isabela Martins. (2017). Uso do Scratch no Brasil com objetivos educacionais: uma revisão sistemática. RENOTE. Revista Novas Tecnologias na Educação. UFGRS, v. 15, p. 1-10.

Kietchenham, B. A. (2007). Guidelines for performing Systematic Literatura Reviews in Software Engineering.

Medeiros, T.J.; Da Silva, T. R.; Aranha, E. H. S. (2013). Ensino de programação utilizando jogos digitais: uma revisão sistemática da literatura. In: Revista Novas Tecnologias na Educação (RENOTE), v.11, n.2.

Scaico, A., Scaico, P. (2016). "Uso de Jogos em Cursos Introdutórios de Programação

no Ensino Superior na Área de Computação: Uma Revisão Sistemática”. In Anais do SBIE 2016, pp. 549-558.

Silva, T. et al. (2014).“Jogos Digitais para Ensino e Aprendizagem de Programação: uma Revisão Sistemática da Literatura”. In Anais do SBIE 2014, pp. 692-701. 